\title{
Schlösser und Herrenhäuser im Wurzener Land
}

\author{
Matthias Donath
}

In den Dörfern um Wurzen sind große und kleine Herrensitze zu finden, sowohl Schlösser von herausragendem kulturgeschichtlichen Interesse als auch Herrenhäuser von eher bescheidenem Umfang. Man kann nicht sagen, dass sich die Schlösser des Wurzener Landes von denen der weiteren Umgebung sonderlich unterscheiden. Gleichwohl sind sie von prägender Bedeutung für die Kulturlandschaft, weil sie in besonderer Weise die Herrschaftsgeschichte des ländlichen Raumes nachzeichnen. Wenn sich dieser Beitrag vornehmlich mit der äußeren Gestalt befasst, dann hat das auch damit zu tun, dass infolge der Enteignung des Jahres 1945 die Ausstattungen der Schlösser die Ahnengalerien, Kunstsammlungen, Bibliotheken und Archive - größtenteils verloren gegangen sind.

Um zu einer geografischen Abgrenzung zu gelangen, wurden diesem Beitrag die Grenzen des Stiftsamtes Wurzen zu Grunde gelegt. Das Stiftsamt Wurzen war Teil des Hochstifts Meißen, des weltlichen Besitzes der Meißner Bischöfe. Der letzte Bischof von Meißen hatte zwar 1581 auf sein Amt verzichtet, doch war das Hochstift als reichsrechtlich eigenständiges Gebilde erhalten geblieben. Die drei dem Hochstift verbliebenen Territorien - das Stiftsamt Wurzen, das Amt Mügeln und das Klosteramt Sornzig - wurden durch eine Stiftsregierung verwaltet, die ihren Sitz in Wurzen hatte. Erst 1818 wurde die Stiftsregierung aufgehoben und das Stiftsgebiet vollständig in das Königreich Sachsen inkorporiert.

Innerhalb des Stiftsamtes Wurzen lagen folgende Rittergüter: Burkartshain, Falkenhain, Großzschepa, Kühnitzsch, Lossa, Müglenz, Mühlbach, Nischwitz, Püchau, Roitzsch, Sachsendorf, Schmölen und Niederschmölen, Thallwitz, Thammenhain, Voigtshain, Wäldgen, Zschochau und Zschorna sowie der Sattelhof Pausitz. In der Exklave bei Belgern lagen die Rittergüter Adelwitz, Ammelgoßwitz und Dröschkau, die hier nicht behandelt werden sollen. Jedes Rittergut war mit einem entsprechend gestalteteten Herrensitz verbunden und verfügte über einen Gutshof. Die Rittergüter hatten sich aus Besitzungen der Gefolgsleute der Bischöfe von Mei-

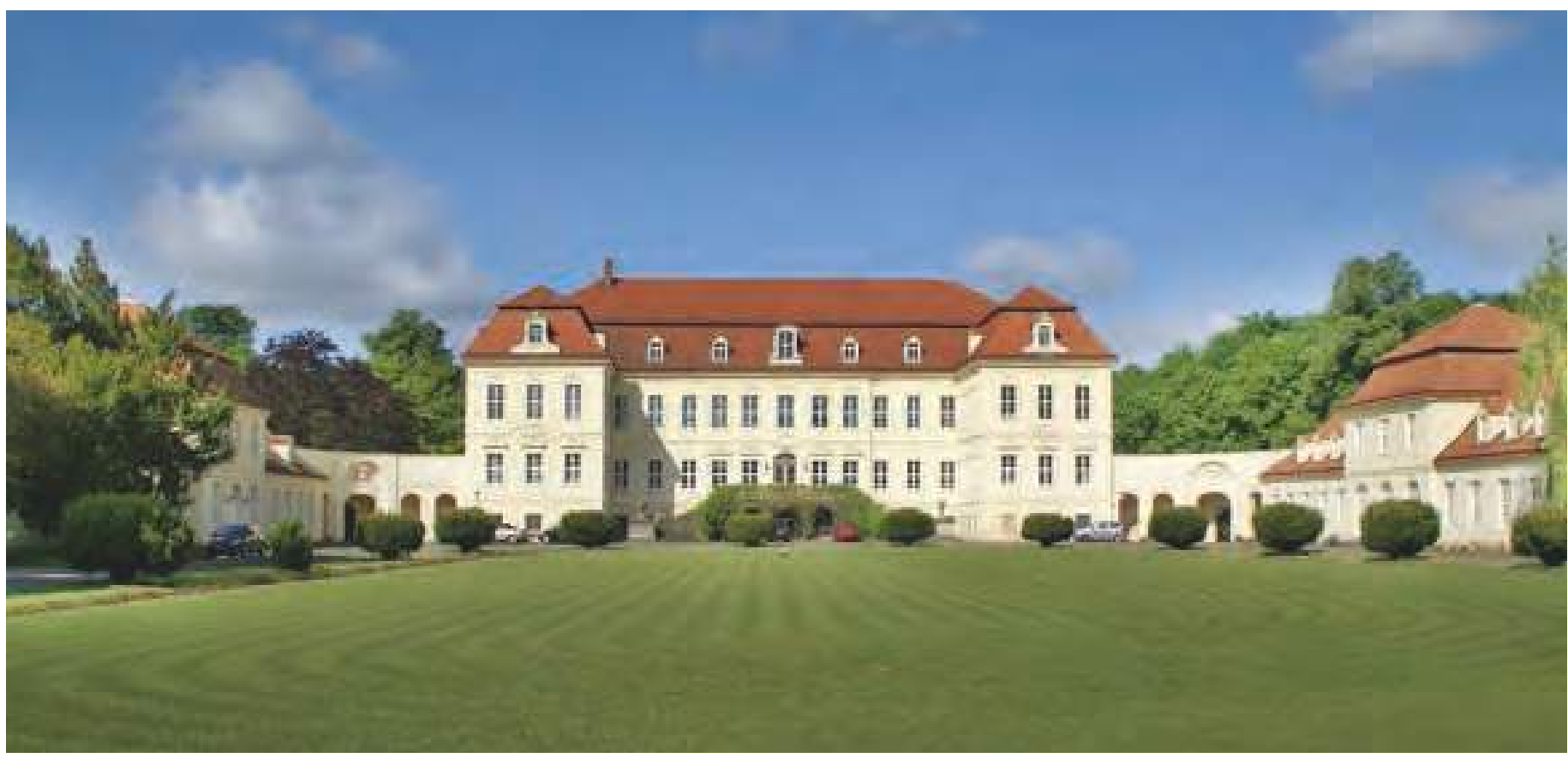


ßen entwickelt. Sie gelangten jedoch schon früh an Adelsfamilien, die aus anderen Landschaften zugewandert waren. Seit dem 17. Jahrhundert lassen sich bürgerliche Rittergutsbesitzer nachweisen. 1581 gingen die weltlichen Herrschaftsrechte des Meißner Bischofs auf Kurfürst August von Sachsen (1526-1586) über, der vom Domkapitel zum Stiftsherrn gewählt wurde. Seitdem stellten die Kurfürsten von Sachsen als Stiftsherren des Hochstifts Meißen die Lehnsurkunden aus.

Der älteste und größte Herrschaftssitz des Wurzener Landes befindet sich links der Mulde in Püchau. Die Geschichte des Herrschaftssitzes reicht bis ins 10. Jahrhundert zurück. Die erste Burg dürfte eine Gründung des deutschen Königs Heinrichs I. (um 876-936) gewesen sein. Der Chronist Thietmar von Merseburg erwähnt zum Jahr 924 den Burgward „Bichni“. Damit ist Püchau der am frühesten bezeugte Ort in Sachsen. Um das hohe Alter Püchaus herauszustellen, brachte man 1850 über dem Portal des Schlossturms eine Sandsteinfigur des deutschen Königs sowie eine Inschriftentafel an, die Thietmar von Merseburg zitiert. Püchau war seit dem 15. Jahrhundert im Besitz der Adelsfamilie Spiegel. Es folgten die von Ende, von Taube und von Bünau, bevor Schloss und Rittergut infolge einer Eheschließung 1807 an die Grafen von Hohenthal gelangten. Diese behielten Püchau bis 1945. Auf die Umgestaltung des Schlosses im späten 19. und frühen 20. Jahrhundert wird noch einzugehen sein.

Mittelalterliche Bausubstanz ist lediglich in Thammenhain erhalten geblieben. Dort befindet sich im Erdgeschoss ein Raum mit einem spätgo-

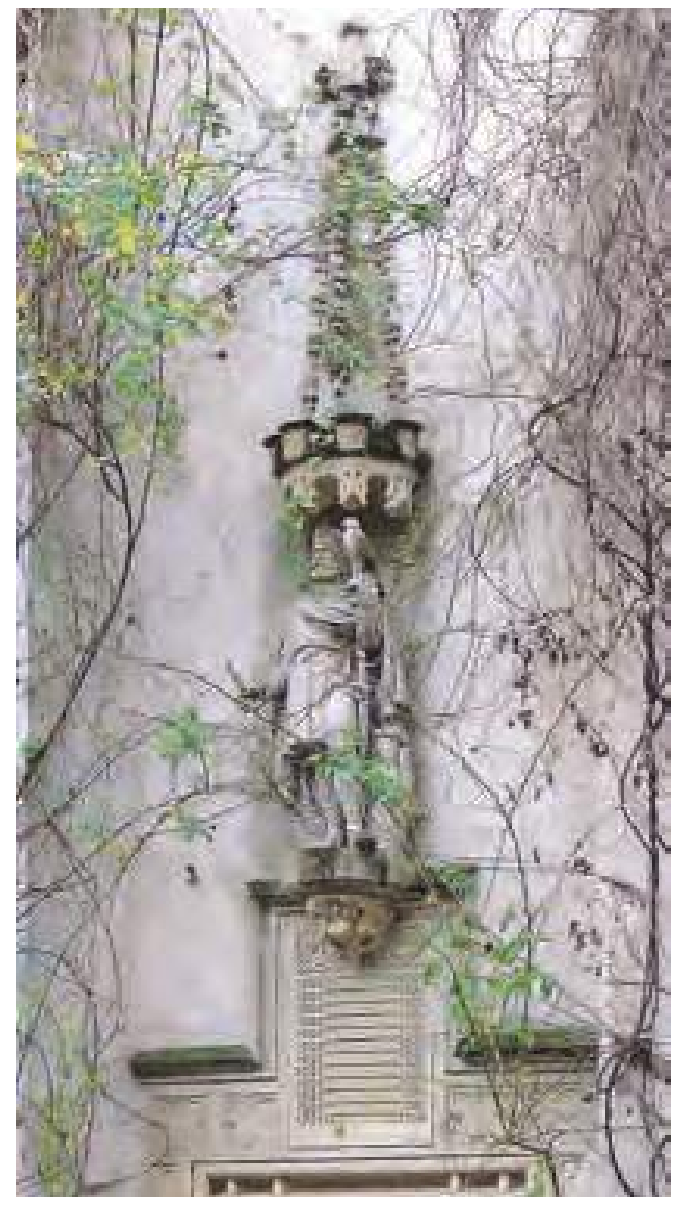

tischen Kreuzrippengewölbe, der in die Jahre um 1480 datiert werden kann. In Thammenhain wurde Johann VI. von Salhausen (1444-1518) geboren, der spätere Bischof von Meißen, wie man seinem Lebenslauf im „Liber Salhusii“ im Domarchiv Meißen entnehmen kann.

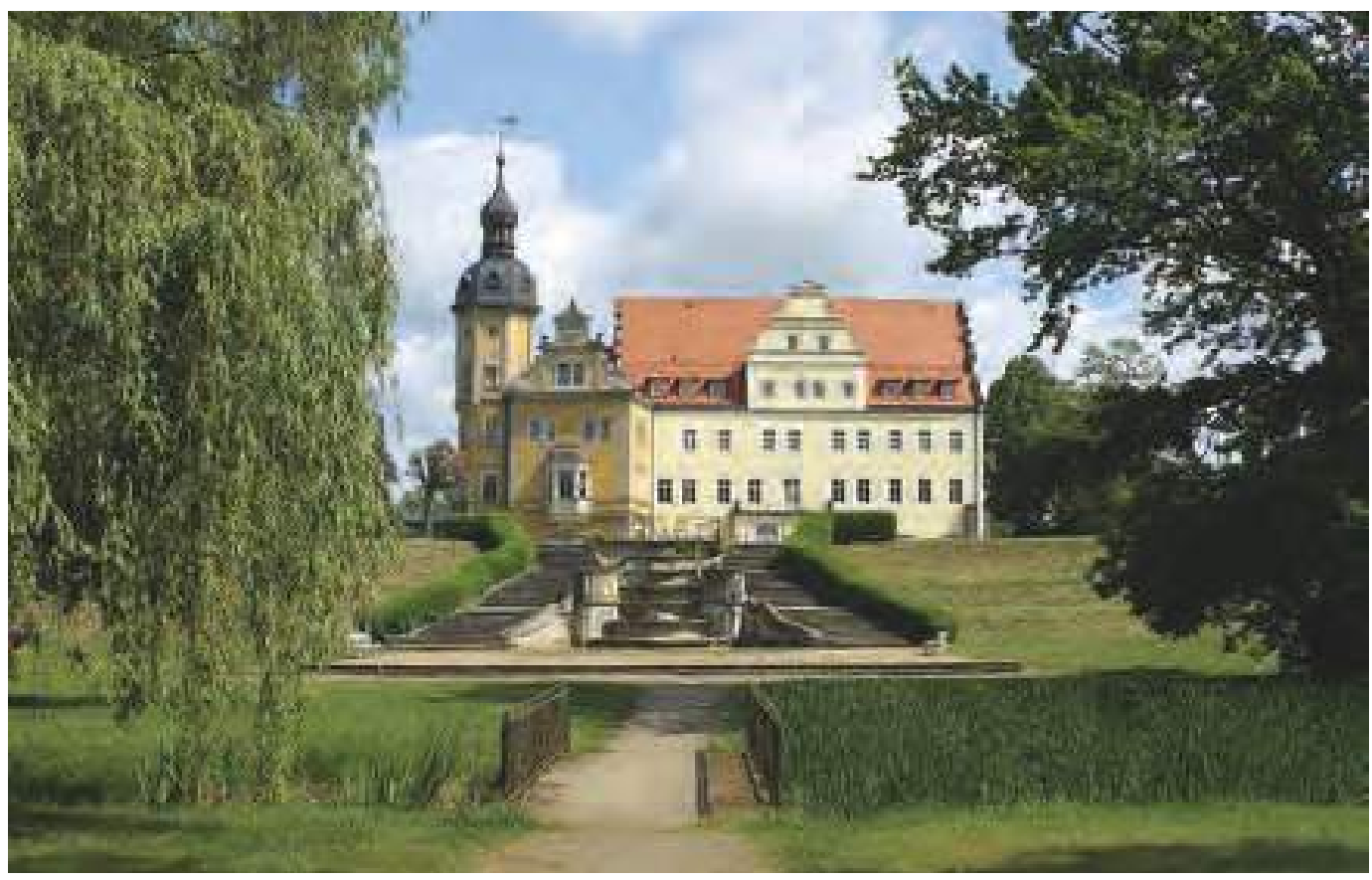

Schloss Thallwitz, Parkseite, rechts der Renaissancebau des 16. Jahrhunderts, links die Ergänzung von 1882 Foto: Jens Ziegenbalg

Schloss Püchau, Statue König Heinrichs I. am Treppenturm Foto: Peter Altmann 
In der Mitte des 16. Jahrhunderts setzte sich der Baustil der Renaissance durch. Die Renaissanceschlösser sind an ihren Ziergiebeln, Erkern und Treppentürmen zu erkennen. Beispiele dieses Baustils sind die Herrenhäuser in Lossa, Falkenhain, Thallwitz und Wäldgen. In Lossa wurde um 1560 durch Moritz von Nitzschwitz ein recht einfaches Bauwerk errichtet. Über den symmetrisch gegliederten Fassaden erhebt sich ein hohes Satteldach, das an den Schmalseiten von Giebeln begrenzt wird. Über dem Mittelrisalit erhebt sich ein geschweifter Renaissancegiebel. Das Herrenhaus in Wäldgen muss den Bauformen zufolge in der Mitte des 16. Jahrhunderts entstanden sein. Möglicherweise wurde es für Hans von Holleufer erbaut, dem auch die Rittergüter Burkartshain und Mühlbach gehörten. Der zweigeschossige Bau wird von einem Zwerchhaus mit spitzem Giebel bekrönt, der durch Lisenen gegliedert wird. Auch das Herrenhaus in Falkenhain ist ein vergleichsweise schlichtes Gebäude. Wohl um 1570 erbaut, hat es einen breitgelagerten rechteckigen Kern, der an den Längsseiten durch Anbauten erweitert wird. Die Giebelaufbauten wurden beseitigt, als man im 18. Jahrhundert eine barocke Bedachung aufsetzte. Weitaus schmuckvoller erscheint da das Schloss in Thallwitz. Um 1580 errichteten die Herren von Canitz das heute noch bestehende Renaissanceschloss. Das giebelgeschmückte Bauwerk erhebt sich über einem einfachen rechteckigen Grundriss. Die Zwerchhäuser, die das steile Dach untergliedern, haben eindrucksvolle Giebelfronten. Auf der Hofseite ist dem Schloss ein Treppenturm mit Wendelstein vorgesetzt, während an der Nordwestecke ein zweigeschossiger Erker das schlichte Fassadenbild belebt.

Im 18. Jahrhundert wurde mehrere Herrensitze im barocken Stil umgebaut oder neu errichtet. Die barocken Herrensitze zeichnen sich durch streng gliederte Fassaden mit gleichmäßig aufgereihten Fensterachsen aus. Der Eingang liegt immer in der Mittelachse. Meist wurde ein hervortretender Mittelrisalit ausgebildet. Größere Schlösser haben auch Seitenflügel, die in symmetrischer Gestalt einen Ehrenhof umgeben.

Die schönste Barockanlage des Wurzener Landes ist in Nischwitz zu finden. Das Schloss ist aus einem älteren Herrenhaus entstanden, das der sächsische Kammerherr und Stallmeister Gustav Carl Freiherr von Rackwitz zwischen 1714 und 1720 erbauen ließ. 1722 verkaufte er das Rittergut an Friederike Charlotte von Wendt, die den Besitz ihrer Tochter Amalie Sophie von Wallmoden (1704-1765), einer Mätresse des britischen Königs Georg II. (16831760), vererbte. Das Herrenhaus und ein Teil der Gutsgebäude waren 1726 abgebrannt, aber gleich wiederaufgebaut worden. 1743 kaufte der sächsische Premierminister Heinrich Graf von Brühl (1700-1763) das Rittergut. Er ließ das ältere Herrenhaus um 1750 durch Johann Christoph Knöffel (1686-1752) zu einer repräsentativen Dreiflügelanlage erweitern und innen neu ausstatten. Die opulenten Wand- und Deckengestaltungen wurden im zweiten Drittel des 18. Jahrhunderts von Dresdner Hofkünstlern geschaffen. Garten- und Festsaal sind mit Stukkaturen sowie mit Wand- und Deckenge-
Herrenhaus Wäldgen, Zustand 2012 Foto: Matthias Donath

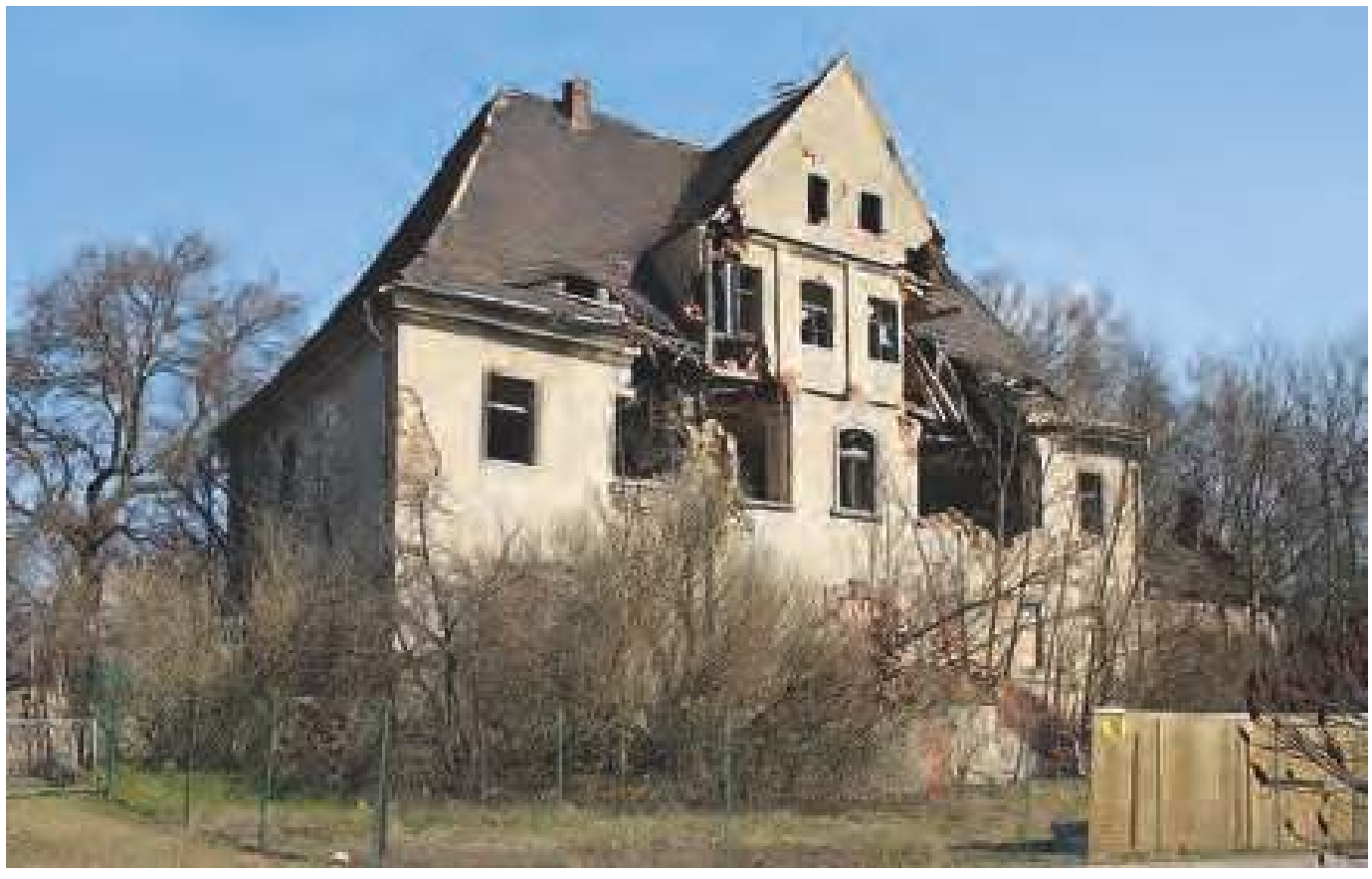




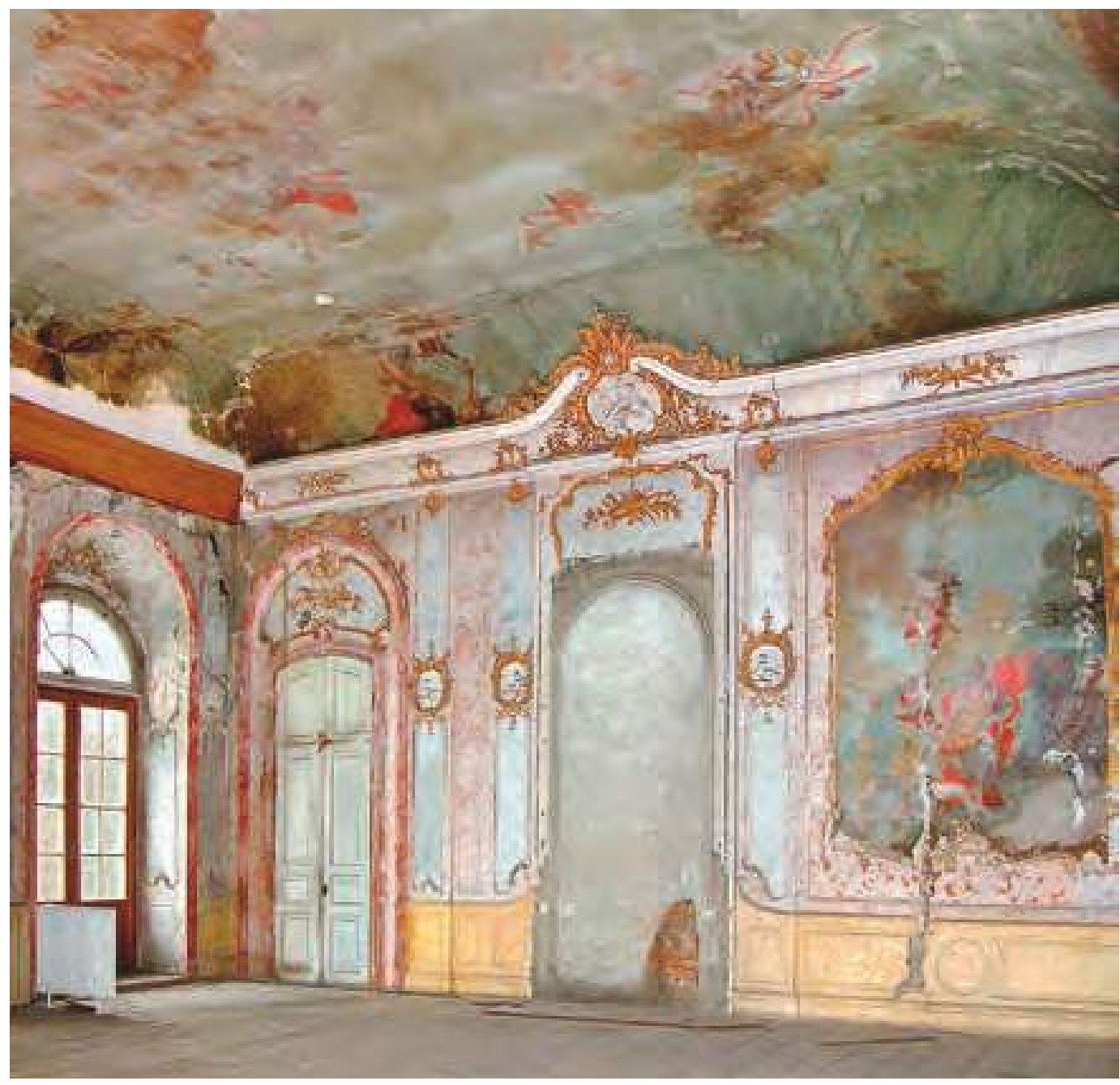

mälden des italienischen Malers Stefano Torelli (1712-1784) ausgekleidet. Friedrich II. von Preußen (1712-1786) hatte einen abgrundtiefen Hass auf den Grafen Brühl. Als preußische Truppen im Siebenjährigen Krieg nach Sachsen einfielen, befahl er ihnen, alle Besitzungen des Grafen Brühl zu plündern und zu zerstören. So wurde Schloss Nischwitz 1758 ausgeraubt und schwer beschädigt. Der Festsaal und die umliegenden Räume waren seitdem kaum mehr in Gebrauch, obwohl das Schloss noch bis 1945 von den Rittergutsbesitzern, zuletzt von der geadelten Familie von Zimmermann, bewohnt wurde.

Eine kleine barocke Dreiflügelanlage wurde in Großzschepa errichtet. Dagegen waren die Schlösser und Herrenhäuser in Hohburg, Voigtshain, Roitzsch und Sachsendorf schlichte Einflügelbauten mit barocker Fassadengestaltung.

Im 19. Jahrhundert wurden zahlreiche Schlösser aufwendig umgestaltet. Ein eindrucksvolles Beispiel ist Püchau, wo die Grafen von Hohenthal einen märchenhaften Mittelaltertraum mit Türmchen, Erkern und neugotischen Maßwerk- fenstern inszenierten. Der neugotische Umbau des Schlosses zog sich über vier Generationen hin. Zunächst überzogen der Leipziger Baumeister Oscar Mothes (1828-1903) und der Berliner Architekt Richard Lucae (1829-1877) die Fassaden mit Stuckdekorationen im englischen Tudorstil, bevor dann der Dresdner Akademieprofessor Constantin Lipsius (1832-1894) 1874/75 den neugotischen Westflügel errichtete. Die zum Hof gerichtete Hauptfront des Schlosses erhielt eine prunkvolle Schaufassade mit Türmen, Maßwerkfenstern, Erkern und Giebeln. Nach einem Sturmschaden 1912 vereinfachte das bekannte Dresdner Architekturbüro Lossow \& Kühne, das sich mit modernen Bauten einen Namen gemacht hatte, die reich gegliederte Dachlandschaft, womit ein Teil des romantischen Bildes verloren ging.

Den Baustil der Neorenaissance vertreten gleich zwei Schlösser. In Thallwitz fügte der Leipziger Architekt Arwed Rossbach (1844-1902) an den Schlossbau des 16. Jahrhunderts 1882 an einen malerisch untergliederten Schlossflügel an, der im Stil der deutschen Neorenaissance mit Erkern und Giebeln dekoriert sowie mit einem
Schloss Nischwitz, Festsaal Foto: Matthias Donath 
Schloss Püchau, Westflügel Foto: Matthias Donath

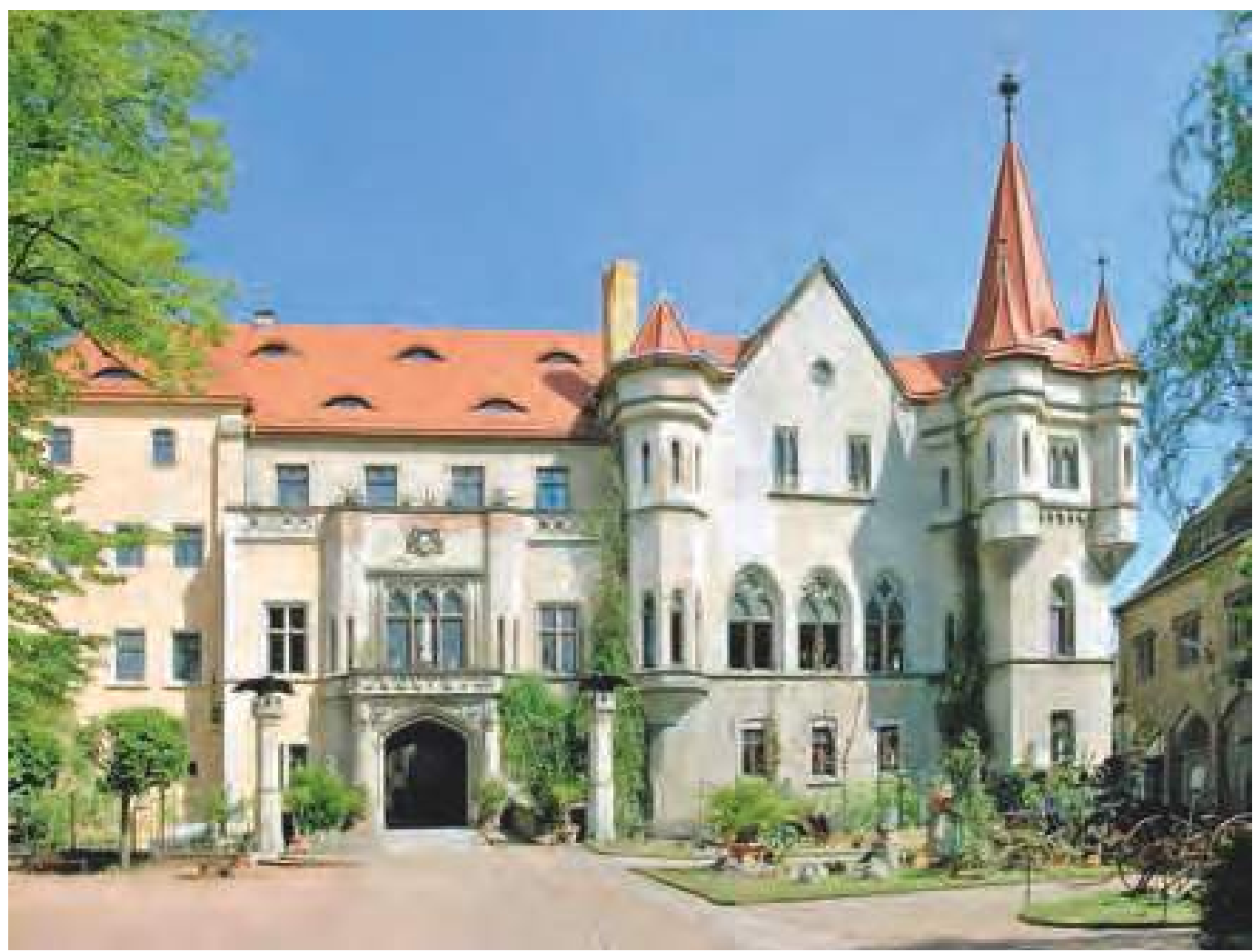

Turm versehen wurde. Thallwitz war Jagdsitz des Fürsten Heinrich XIV. Reuß (1832-1913), der das Fürstentum Reuß jüngere Linie regierte. Das Rittergut Thallwitz war bereits Anfang des 19. Jahrhunderts durch eine Heirat an das Fürstenhaus gelangt. 1890/91 ließ Adolf von Schönberg das Schloss in Thammenhain, einen schlichten Bau des 17. Jahrhunderts, mit einer Bekleidung im Stil der deutschen Neorenaissance versehen. Der Architekt Adolf Leyn aus Hannover schmückte den Bau mit Erkern, Giebeln und verschieden geformten Fenstern. Auf der Südseite wurde ein Turm vorgesetzt. Durch den Haupteingang auf der Nordseite

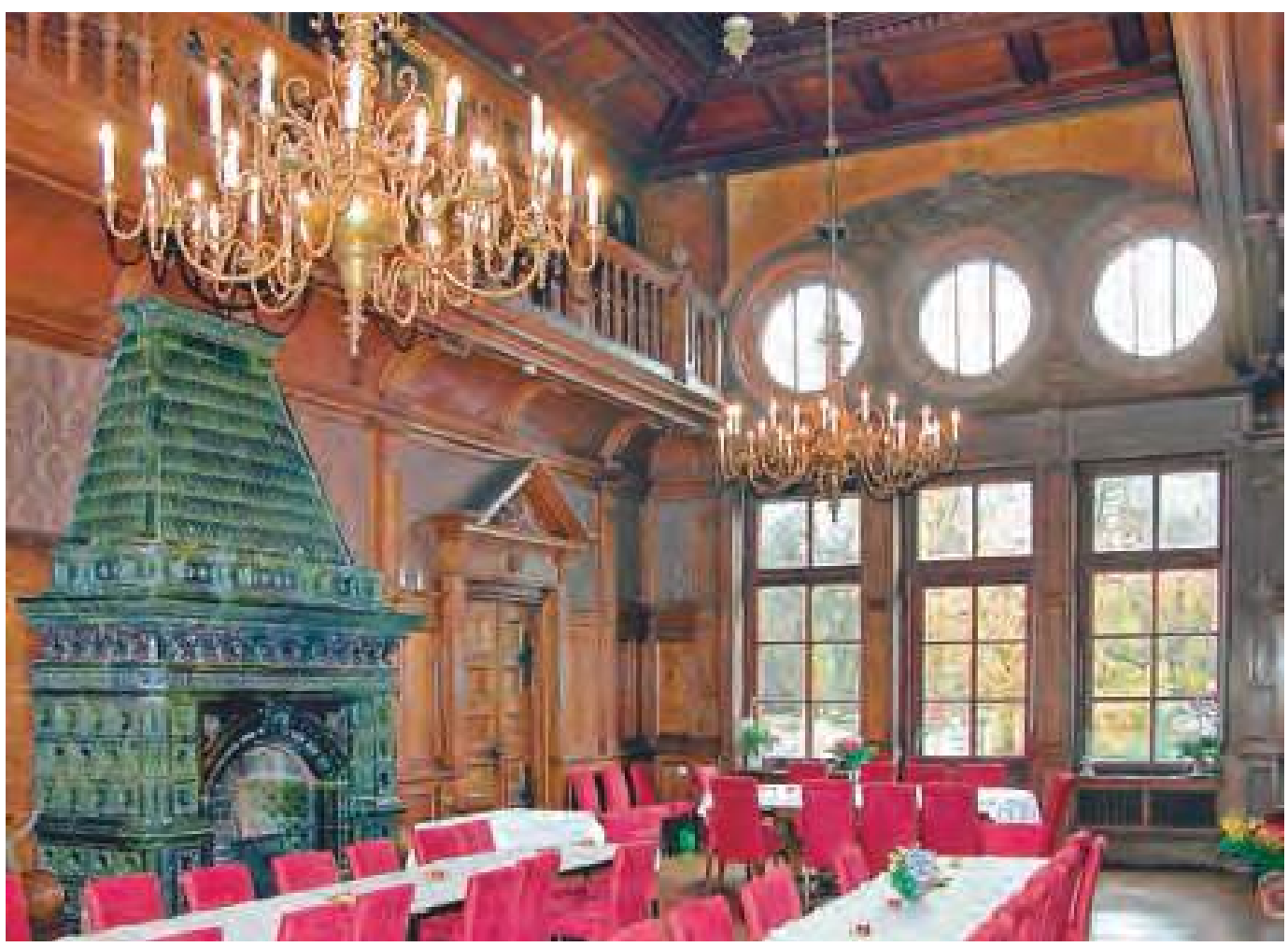




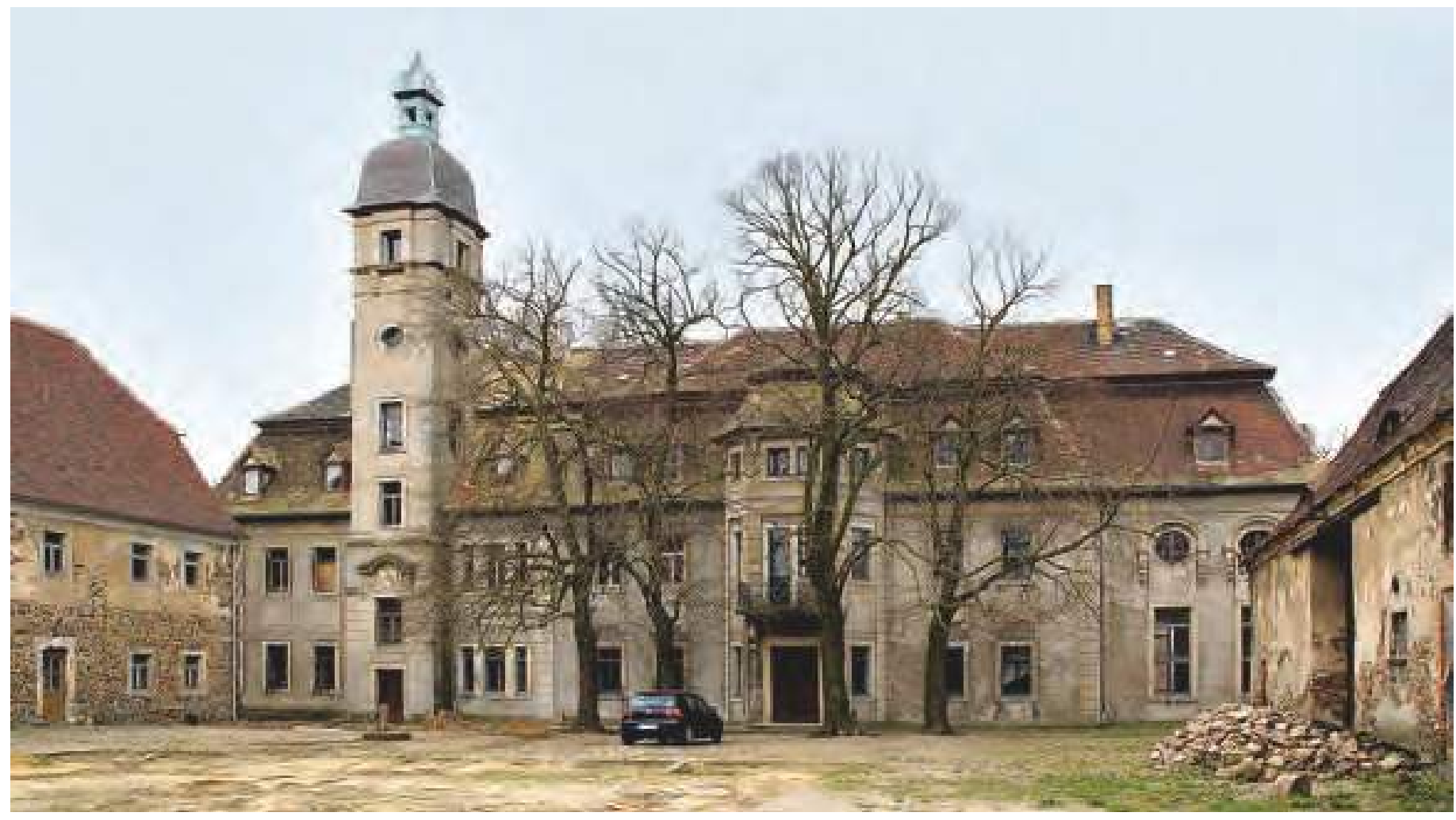

gelangt man in ein geräumiges Vestibül. Es schließt sich ein beeindruckender, durch zwei Stockwerke reichender Saal an. Er ist im altdeutschen Stil ausgestattet und über eine große Fensterfront zum Park geöffnet. Die Eichenholztäfelungen schuf Franz Schneider aus Leipzig. Zur historischen Ausstattung gehören zwei Kronleuchter der Wurzener Bronzefabrik und ein aus grün glasierten Kacheln gesetzter Ofen. Der Umgang im zweiten Stockwerk dient als Ahnengalerie. Hier hängen Ahnenbilder der Familie von Schönberg aus dem 16. bis 19. Jahrhundert. Sie waren nach 1945 durch die "Schlossbergung" in Museen und Depots gelangt, wurden aber 2005 an Dr. Rüdiger Freiherr von Schönberg zurückerstattet.

Das Schloss in Müglenz erhielt erst zu Beginn des 20. Jahrhunderts seine eindrucksvolle Gestalt. 1905/06 ließ Georg Nette, ein bürgerlicher Rittergutsbesitzer, das alte Herrenhaus des 18. Jahrhunderts an beiden Schmalseiten verlängern. Der namentlich nicht bekannte Architekt setzte zwar barocke Stilmittel ein, vermied es aber, klare Symmetrien auszubilden. Er nahm stattdessen eine malerische Gliederung vor, die die strenge Ordnung aufbricht und belebt. Auf der Gartenseite wurden eine Giebelachse geschaffen, ein Erker mit Kupferdach vorgesetzt und ein Wintergarten mit Balkon errichtet, doch diese baulichen Glieder sind so verteilt, dass sich kein gleichmäßiges Fassadenbild ergibt, wie man es bei Barockschlössern erwar- tet. Auch der Schlossturm auf der Hofseite wurde außermittig angeordnet. Seine baulichen Einzelheiten sind barocken Kirchtürmen nachgebildet. Auch das Schloss im nahen Zschorna ist durch eine Mischung aus historischen und modernen Elementen geprägt. Es wurde 1904/05 für den Leipziger Fabrikanten Curt Jacob außerhalb des alten Rittergutshofs neu erbaut. Während die Giebel auf Vorbilder des 17. Jahrhunderts zurückgehen, sind die übrigen Bauformen durch den Reformstil beeinflusst, der vor dem Ersten Weltkrieg im Mode war.

Die Geschichte der Rittergüter in Sachsen endete nach dem Zweiten Weltkriegs mit einem radikalen Bruch: Mit Unterstützung der sowjetischen Besatzungsmacht setzten deutsche Kommunisten eine Enteignung des Großgrundbesitzes und eine Umverteilung des Grund und Bodens durch. Alle landwirtschaftlichen Betriebe, die eine Größe von über 100 Hektar hatten, wurden ohne Entschädigung enteignet. Im Wurzener Land waren alle Rittergüter betroffen. Gegen die teils adligen, teils bürgerlichen Rittergutsbesitzer, die allesamt als "Junker" beschimpft wurden, richtete sich blinder Hass. Man verhaftete die Enteigneten und in „Sippenhaft" auch ihre nächsten Angehörigen. Die Gutsbesitzer wurden auf die Burg Colditz in ein Internierungslager gebracht. Von dort erfolgte die Deportation in Viehwaggons - nicht nach Sibirien, wie die Betroffenen befürchten mussten, sondern auf die Insel Rügen. Mehrere Ent-
Schloss Müglenz, Hofseite Foto: Matthias Donath 
Rittergut Thammenhain, Abbruch der Wirtschaftsgebäude und der Hofkirche (rechts), 1949 Foto: Rüdiger Freiherr von Schönberg

Schloss Lossa mit neuem Dach, aber ohne Nutzung Foto: Matthias Donath

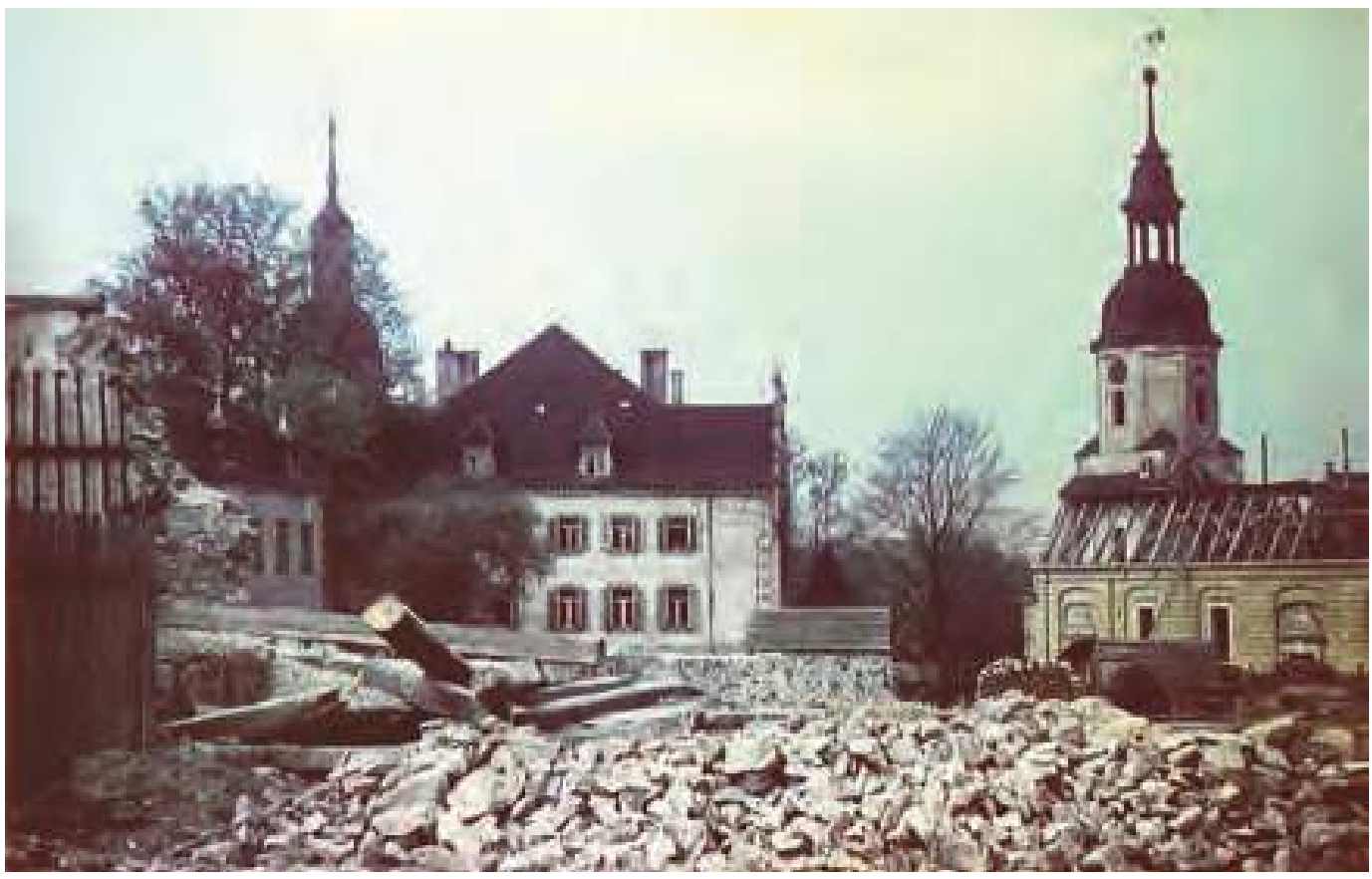

eignete starben an Hunger und Entkräftung. Die meisten Deportierten konnten im Winter 1945/45 von der Insel Rügen fliehen. Sie entkamen in die westlichen Besatzungszonen und versuchten dort, ein neues Leben $\mathrm{zu}$ beginnen. Unterdessen wurden die Schlösser geplündert und das Inventar abtransportiert.

Nach dem Befehl 209 der sowjetischen Besatzungsmacht vom 9. September 1947 sollte das Baumaterial für die Errichtung von Neubauernhöfen durch den Abbruch von Schlössern und Herrenhäusern gewonnen werden. Mit der Beseitigung der „Zwingburgen des Junkertums“ wollte man die Erinnerung an die Rittergüter und ihre Besitzerfamilien auslöschen. Es war reine Willkür, welche Schlösser und Herrenhäuser von der Landesbodenkommission zum Abbruch freigegeben wurden. $\mathrm{Zu}$ den Verlusten im Wurzener Land gehören das Schloss in Sachsendorf sowie die Herrenhäuser in Hohburg, Niederschmölen und Pausitz. Auch wurde in Thammenhain die evangelische Schlosskirche abgebrochen, die seit dem 18. Jahrhundert zum Schlossensemble gehört hatte.

In den Jahren der DDR wurden die Schlösser zu unterschiedlichen Zwecken genutzt, aber kaum

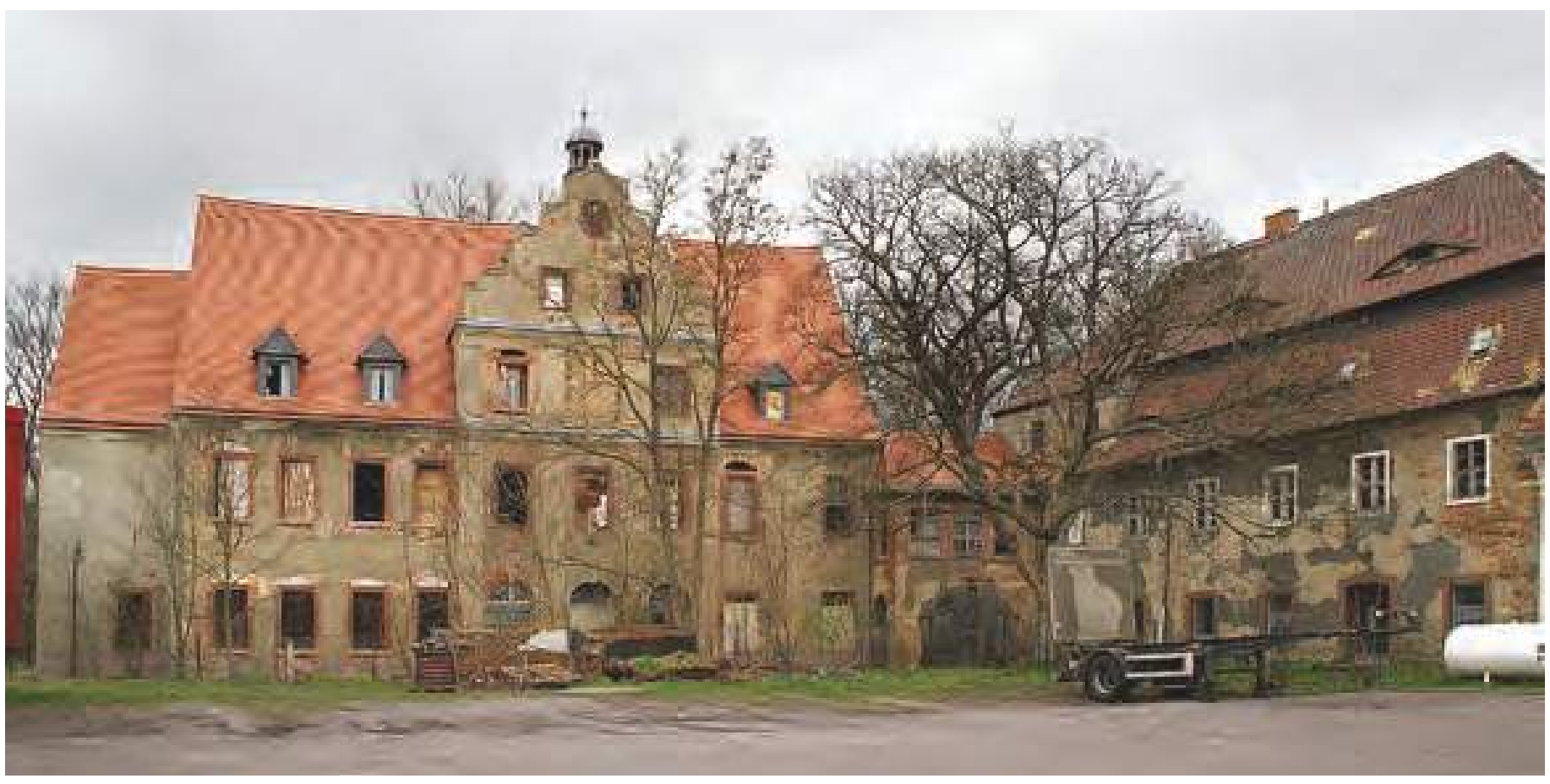




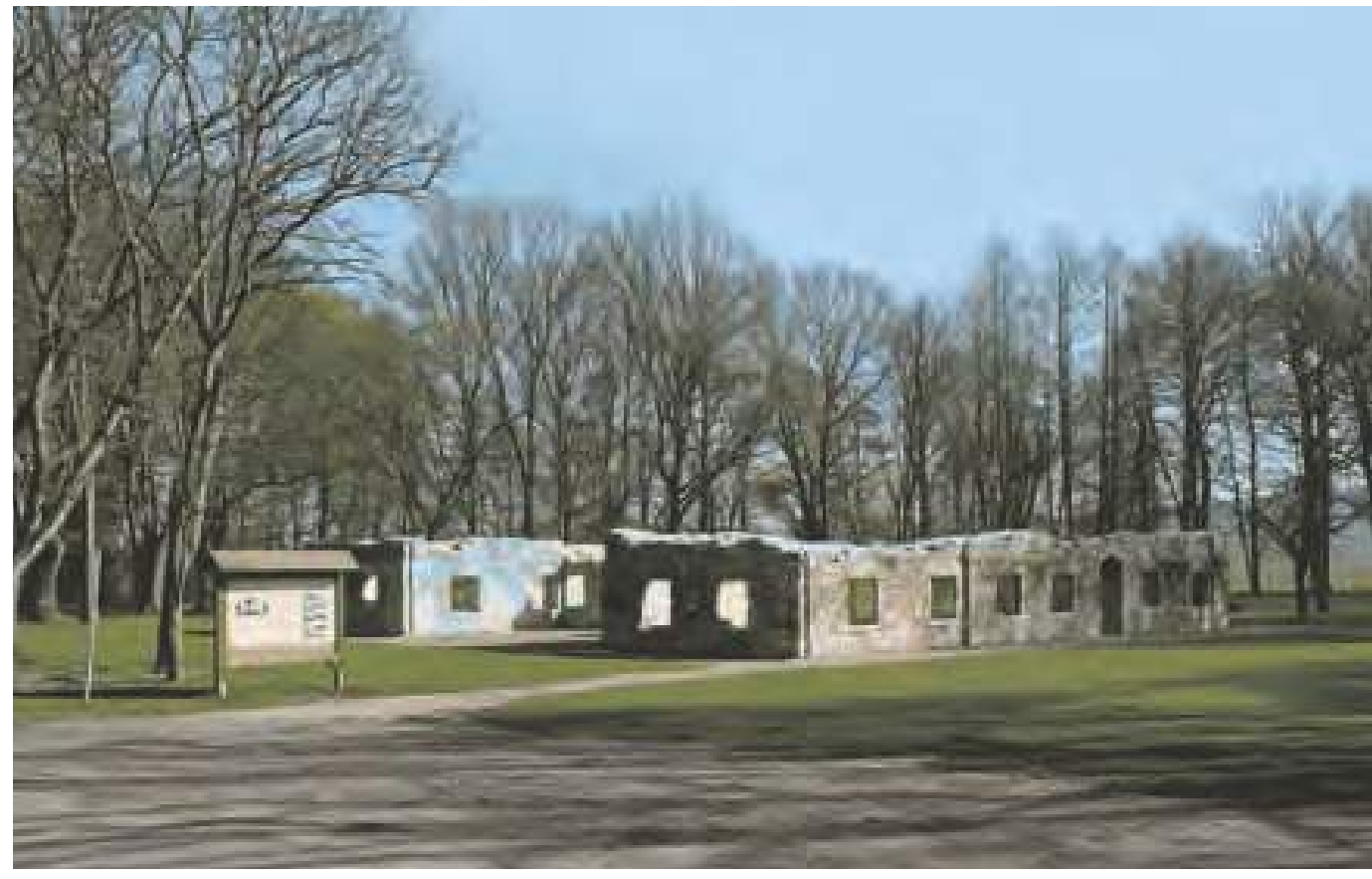

baulich unterhalten. Eine Ausnahme bildete das Schloss Nischwitz, das in den 1980er Jahren in den Blick der staatlichen Denkmalpflege geriet. Nach dem Ende der DDR hat sich die Situation teils verbessert, teils verschlechtert. Die Entscheidung, die Enteignungen der Jahre 1945 bis 1949 nicht rückgängig zu machen, verhinderte eine Wiederbelebung der Schlösserlandschaft. $\mathrm{Da} \mathrm{zu}$ den Gebäuden heute kein Landwirtschaftsbetrieb mehr gehört, fehlt die wirtschaftliche Grundlage zu ihrer Unterhaltung. Immerhin haben sich zwei Familien aus emotionalen Gründen dazu durchgerungen, das Haus ihrer Vorfahren zurückzukaufen. In Thammenhain erwarb Dr. Rüdiger Freiherr von Schönberg das Schloss, in dem er selbst seine ersten Lebensjahre verbracht hatte und das seit 1947 als Kinderheim und später Altersheim der Caritas genutzt worden war. Er und sein Bruder haben auch Wald- und Ackerflächen in der Umgebung erworben. In Mühlbach kaufte der Landwirt Dieter Jung das Rittergut zurück, das seinen Vorfahren in drei Generationen gehört hatte. Aufgrund eines Vergleichs, in dem es um eine Entschädigung für zu Unrecht enteignete Vermögenswerte des Fürstenhauses Reuß ging, wurden Schloss und Park in Thallwitz 2008 an Woizlawa-Feodora Prinzessin Reuß (geb. 1918) zurückübertragen.

Die Schlösser in Falkenhain, Voigtshain, Müglenz, Kühnitzsch, Schmölen und Püchau sind in private Hand übergegangen. Die Eigentümer verfolgen unterschiedliche Konzepte und sind bei der Wiederbelebung und Restaurierung der Herrensitze unterschiedlich weit vorangeschritten.
Auf der anderen Seite sind viele Herernhäuser dem Verfall preisgegeben. Sie gelangten an Eigentümer, die keine Verwertungsmöglichkeit sahen und nichts unternahmen, um das Erbe zu erhalten. Besonder schlimm sieht es in Wäldgen aus, wo Teile des Giebels und des Daches eingestürzt sind. Eine Rettung scheint kaum noch möglich zu sein. In Lossa erfolgte zwar eine Notsicherung mit Mitteln der Deutschen Stiftung Denkmalschutz, doch gelang es der Gemeinde nicht, den weitgehend entkernten Herrensitz an einen neuen Eigentümer zu verkaufen. Die Herrenhäuser in Burkartshain und Zschorna stehen leer.

Eine tragische Entwicklung vollzog sich in Großzschepa. Noch in den 1990er Jahren war das Schloss weitgehend intakt. 2002 gelangte der Herrensitz an einen Eigentümer, der nach Abnahme der Dachdeckung mit einer Reparatur des Dachtragwerks beginnen wollte. Da er diese Baumaßnahme jedoch ohne Genehmigung begonnen hatte, wurde ein Baustopp ausgeprochen und ein Zwangsgeld durch das Landratsamt angedroht. Damit wurde die Bautätigkeit eingestellt. Da das Schloss nunmehr ohne Dach dastand, kam es zu einem rapiden Verfall. Leider war es nicht möglich, mittels einer Ersatzvornahme eine Notsicherung durchzuführen, die das Baudenkmal noch gerettet hätte. Die Behörden nahmen sehenden Auges den Untergang des Schlosses in Kauf. Schließlich sah die Gemeinde Lossatal keine Alternative mehr, als den verwahrlosten „Schandfleck“ abzutragen. Heute stehen von der Dreiflügelanlage nur noch die Umfassungsmauern des Erdgeschosses. Hoffen wir, dass zu diesem Verlust in den nächsten Jahren nicht noch weitere hinzukommen.
Schloss Großzschepa, Zustand nach Abbruch mit Resten des Erdgeschosses Foto: Jürgen Schmidt

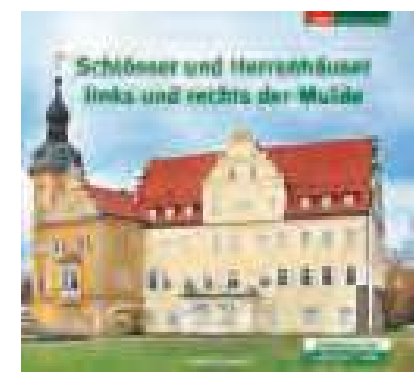

Zum Weiterlesen: Matthias Donath: Schlösser und Herrenhäuser links und rechts der Mulde (Schlösser im Leipziger Land 1). Meißen 2012

Zu beziehen über: Redaktions- und Verlagsgesellschaft Elbland Niederauer Straße 43 01662 Meißen Tel.: 03521-41045520 E-Mail: sz.meissen@dd-v.de 